\title{
Detecting Accuracy of Three Dimensional Power Doppler (3DPD) Vascular Indices for Prenatal Diagnosis of Morbidly Adherent Placenta in Patients with Placenta Previa
}

\author{
Ahmed Sherif Abdel-Hamid', Mohamed Sayed Aly1, Shahira Zakaria Mohamed Ali Ghaly², \\ Maged Mahmoud Elshourbagy ${ }^{1}$
}

${ }^{1}$ Department of Obstetrics and Gynecology, Faculty of Medicine, Ain-Shams University, Cairo, Egypt

${ }^{2}$ Department of Obstetrics and Gynecology in Shoubra General Hospital, Cairo, Egypt

Email:ahmedgyna@yahoo.com

How to cite this paper: Abdel-Hamid, A.S., Aly, M.S., Ghaly, S.Z.M.A. and Elshourbagy, M.M. (2020) Detecting Accuracy of Three Dimensional Power Doppler (3DPD) Vascular Indices for Prenatal Diagnosis of Morbidly Adherent Placenta in Patients with Placenta Previa. Open Journal of Obstetrics and Gynecology, 10, 49-64. https://doi.org/10.4236/ojog.2020.101005

Received: November 13, 2019

Accepted: January 3, 2020

Published: January 6, 2020

Copyright $\odot 2020$ by author(s) and Scientific Research Publishing Inc. This work is licensed under the Creative Commons Attribution International License (CC BY 4.0).

http://creativecommons.org/licenses/by/4.0/

(c) (i) Open Access

\begin{abstract}
Objective: The study's objective was to assess the accuracy of using prenatal 3-dimensional power Doppler analysis of vascular placental indices to accurately diagnose morbidly adherent placenta objectively. Background: Traditionally, 2D ultrasound was used for the diagnosis of a suspected morbidly adherent placenta (MAP) previa. More objective techniques like 3D power Doppler haven't been well studied. Study Design: A prospective cohort study is designed for women with gestational age between 28 and 32 weeks with suspected placenta previa. Patients were examined by $2 \mathrm{D}$ ultrasound which was used in management decisions. 3D Power Doppler's VI, FI and VFI were measured during the same examination after manual tracing of placenta; data were blinded to obstetricians. Histopathology was performed to confirm MAP. Results: Our results showed that the 3D power Doppler VI $\geq 16$ predicted the diagnosis of MAP with $100 \%$ sensitivity, $100 \%$ specificity which is better than those of 2D ultrasound. While VI $>33.1$ measured by 3D Doppler predicted severe MAP with a sensitivity of $73.9 \%$ and specificity of $86.4 \%$, which was superior to $2 \mathrm{D}$ ultrasound. Conclusion: In patients with placenta previa, the 3D Doppler's vascular index accurately predicts MAP. Furthermore, vascular and vascular flow indices of 3D Doppler were more predictive of severe cases of MAP compared to 2D ultrasound.
\end{abstract}

\section{Keywords}

3D Color Doppler, 2D Ultrasound, Placental Vascular Indices, Morbidly Adherent Placenta, Placenta Previa 


\section{Introduction}

Normally, the placenta attaches to the wall of the uterus, however, sometimes the placenta invades deeply into the uterine wall that part or all of the placenta remains attached [1]. Recently, the term "morbidly adherent placenta" (MAP) has been more frequently used to describe abnormal implantation of the placental villi into the uterine wall [2]. It is histopathological term-occurs when the placenta fails to detach from the wall of the uterus due to abnormal implantation at the basal plate [3].

Approximately 1 out of every 533 pregnancies, according to the American College of Obstetricians and Gynecologists (ACOG) experience placenta accrete, percreta or increta [3]. The difference between percreta, increta or accreta which are the pathological types used to describe placental invasion, is determined by how deep the placental villi invade into the uterine wall [4] [5] [6].

There is a systematic review showed a wide heterogeneity in terminology used to describe the grades of accreta placentation using the $2 \mathrm{D}$ parameters. The authors concluded that the evaluation of the accuracy of ultrasound imaging in screening and diagnosing placenta accreta is limited [5]. In the same review, when combining all cases of placenta accreta, increta and percreta, the loss of sonolucency was the most common finding [5].

Several studies used the 3D color Doppler techniques to diagnose MAP and reported a sensitivity ranging between $39 \%$ and $100 \%$ based on abnormal vascular patterns [7] [8]. In these studies, the placenta was assessed subjectively for abnormal vascularity using the 3D power Doppler technique without any quantification. Shih et al. made the diagnosis of placenta accreta when intraplacental hyper vascularity, inseparable cotyledonal (fetal) and intervillous (maternal) circulations, or tortuous vascularity was noted [7]. Hyper vascularity of the uterine serosa-bladder wall interface and tortuous confluent vessels across the placental width were used as diagnostic criteria for morbidly adherent placenta by Collins et al. [8].

A recent study quantified the amount of vessels involved in the diagnosis of morbidly adherent placenta [9]. However, this was applied to the utero-placental interface only and not the entire placenta. They measured the largest area of confluent 3D power Doppler signal [9]. Although abnormal vascularity of the utero-placental interface is highly suggestive of abnormal placentation, abnormal vessels running throughout the placenta could be missed using this particular technique. In addition, choosing the most confluent area is operator dependent and other areas might have been missed due to subjectivity of the operator's assessment [10].

\section{Aim of the Work}

The study's objective was to assess the accuracy of prenatal 3-dimensional power Doppler to predict the morbidly adherent placenta objectively. 


\section{Patients and Methods}

This was a prospective cohort study conducted between September 2018 and September 2019 at the Departments of Obstetrics and Gynecology, Ain Shams University Maternity Hospital.

- Inclusion criteria: women who were referred to the ultrasound for the re-evaluation of placenta previa and morbidly adherent placenta between 28 and 32 weeks will be included.

- Exclusion criteria: Fetal anomalies and multiple gestations.

Combination of trans abdominal and transvaginal ultrasound performed to confirm the location of placenta using routine 2D imaging using Voluson E8 GE system equipped with $4-8 \mathrm{MHz}$ transducers. Uniform diagnostic criteria were applied to diagnose the suspected MAP using at least one of the following findings:

1) The presence of hypo echoic areas in the body of the placenta (placental lacunae).

2) The loss of the normal hypo echoic myometrium adjacent to the base of the placenta (loss of sonolucency).

3) Absent or disrupted hyper echoic line separating the uterus from the urinary bladder (abnormal uterine serosa-bladder line).

They have all been identified as markers of MAP on 2D ultrasound [2].

Pregnant women with at least one identified 2D findings, underwent an additional imaging using 3D power Dopplertrans abdominal ultrasound of the placenta. After full bladder confirmation, obtained using a GE Voluson E8 RAB4-8 transducer probe this technique was previously described for evaluation of placental vascularity [11] [12].

The study was approved by the Ethical Committee of Faculty of Medicine, Ain-Shams University. Verbal consent is taken from all the enrolled patients after explaining the aim of the study and was voluntarily participated when it was clear that it was an observational study that has no harm to them or to their babies.

The placental images were optimized for every patient to visualize the maximum thickness of the placenta with the suspected MAP at the center of the imaging area. Histogram analysis was applied to the taken images. The 3D placental volumes were assessed by manual tracing at $30^{\circ}$ angle increments to include the maximum viewed placenta. The vascularization index (VI; which is calculated by dividing colored/total voxels, voxels are the cubes that occupy the volume of interest, which is in this case the placenta), the flow index (FI; the average of the color value of all blood flow or the mean intensity of the colored voxels), and the vascular flow index (VFI; is obtained by multiplying VI and FI and dividing the result by 100) which is calculated using the same software. Data was blinded to the maternal fetal medicine specialist interpreting the $2 \mathrm{D}$ ultrasound findings and making recommendations for the decision making of the patient's management of care. $2 \mathrm{D}$ results were blinded too to the sonographer performing 
the 3D examination and calculating VI, FI and VFI.

All patients were delivered by caesarean section followed by hysterectomy (cesarean hysterectomy) as planned by the operative physician-based on combination of suspected diagnosis from $2 \mathrm{D}$ ultrasound and clinical assessment which were used as tools to make the decision before surgery.

If $2 \mathrm{D}$ findings were not suggestive of MAP, hysterectomy was performed when the placental invasion was obvious with increased vascular markings and visualization of placenta through the uterine window, when the placenta failed to separate spontaneously or when there is resistance to manual placental separation with or without hemorrhage was encountered.

The final diagnosis of MAP for the analysis in this study was made based on the pathological examination of hysterectomy specimen by a histo-pathologist.

The patients were divided according to the collected data into 2 groups (group A and group $B)$.

$>$ Group (A): patients without MAP.

- Group (B): patients with MAP confirmed by histopathological examination of hysterectomy specimen and according to this examination they were sub grouped into MAP and severe MAP.

Severe MAP (sMAP) which was identified for this study by the presence of the following components:

* Increta or percreta placenta on histopathology.

* Transfusion of $\geq 2$ units of PRBCs and estimated blood loss $>2000 \mathrm{ml}$.

Blood loss $>2000 \mathrm{ml}$ was considered severe based on previous reviews [13] [14].

\section{Statistic Analysis Method}

Descriptive statistics were done for quantitative data as minimum \& maximum of the range as well as mean $\pm \mathrm{SD}$ (standard deviation) for quantitative normally distributed data, median and 1st \& 3rd inter-quartile range for quantitative non-normally distributed data, while it was done for qualitative data as number and percentage.

Inferential analyses were done for quantitative variables using Shapiro-Wilk test for normality testing, independent $\mathrm{t}$-test in cases of two independent groups with normally distributed data and, ANOVA test with for more than two independent groups with normally distributed data and Kruskal Wallis test with non-normally distributed data. In qualitative data, inferential analyses for independent variables were done using Chi square test for differences between proportions and Fisher's Exact test for variables with small expected numbers as well as Kappa and test for agreement between paired categorical data.

$\mathrm{K}$ values $>80 \%$ were considered very good agreement [15]. Post hoc Bonferroni test was done to find homogenous groups in significant tests among more than two independent groups. ROC curve used to assess the performance of different tests and differentiate between certain groups. The level of significance at 
$\mathrm{p}$ value $<0.050$ considered significant, otherwise results is non-significant.

\section{Results}

100 pregnant patients were included in our study (Figure 1). All cases were delivered by C.S $97 \%$ of them underwent LUS C.S. Among MAP group 6 cases suffered from visceral injuries and 2 cases were admitted to the ICU with mean estimated blood loss of $2.4 \pm 1.3 \mathrm{~L}$ (Table 1).

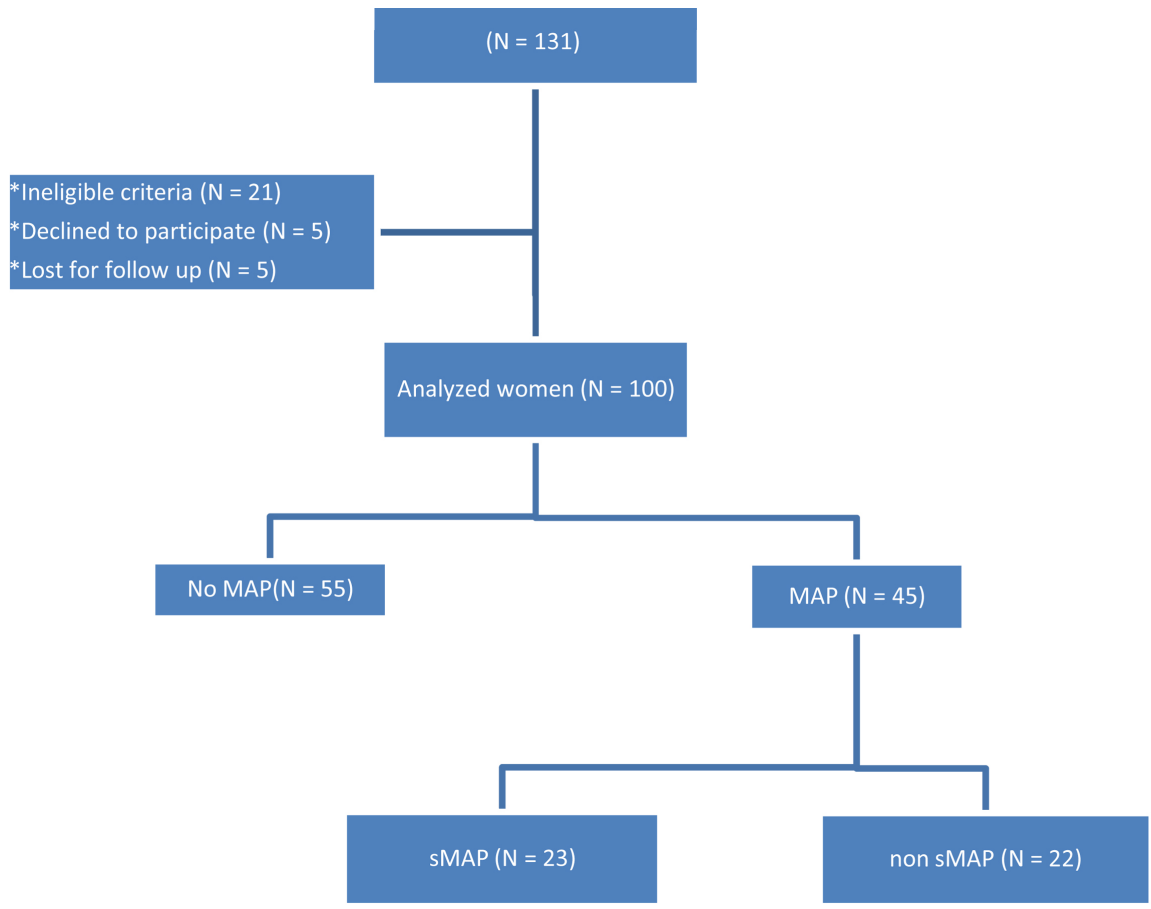

Figure 1. Flow chart of the studied cases.

Table 1. Characteristics and outcomes in MAP vs. no MAP.

\begin{tabular}{lccc}
\hline \multicolumn{1}{c}{ Characteristics } & MAP $(\mathbf{N}=45)$ & No MAP $(\mathrm{N}=55)$ & $\mathbf{p}$ \\
\hline Age (years) & $30.8 \pm 7.0$ & $31.2 \pm 4.0$ & ${ }^{\wedge} 0.751$ \\
GA at investigation (weeks) & $33.8 \pm 3.0$ & $34.8 \pm 1.1$ & ${ }^{\wedge} 0.100$ \\
GA at delivery (weeks) & $35.1 \pm 2.8$ & $35.9 \pm 1.0$ & ${ }^{\wedge} 0.092$ \\
Parity & $3.0(2.0-4.0)$ & $3.0(2.0-3.0)$ & 0.643 \\
Previous CD & $2.0(1.0-3.0)$ & $1.0(0.0-3.0)$ & $0.002^{*}$ \\
HTN & $4(8.9 \%)$ & $1(1.8 \%)$ & ${ }^{\S} 0.171$ \\
DM & $0(0.0 \%)$ & $1(1.8 \%)$ & ${ }^{\S} 1.000$ \\
DC & $6(13.3 \%)$ & $1(1.8 \%)$ & $\$ 0.043^{*}$ \\
Myomectomy & $2(4.4 \%)$ & $0(0.0 \%)$ & ${ }^{\S} 0.200$ \\
Blood loss (L) & $2.4 \pm 1.3$ & $0.8 \pm 0.3$ & ${ }^{\wedge}<0.001^{*}$ \\
Blood transfusion & $(2-12$ unit) & $(1-2$ unit $)$ & $<0.01$ \\
Intra-operative injuries & 6 & 1 & $<0.01$ \\
ICU admission & 2 & 0 & $<0.01$ \\
\hline
\end{tabular}

${ }^{\wedge}$ Independent t-test. ${ }^{\circ}$ Mann Whitney test, ${ }^{*}$ Chi square test. ${ }^{\circledR}$ Fisher's Exact test. ${ }^{*}$ Significant, GA; gestational age, DM: diabetes mellitus, HTN: hypertension, DC: dilatation and curettage, ICU: Intensive care unit. 
Of 33 cases were clinically diagnosed with MAP based on 2D parameters before the delivery even; 27 patients of them underwent a cesarean hysterectomy and were confirmed MAP by histopathology. Among the remaining 6 cases, four had a cesarean delivery without hysterectomy because the placenta separated easily intraoperatively and the remaining two cases had a cesarean hysterectomy with no histological evidence of MAP (both patients suffer of severe uncontrollable intraoperative bleeding).

And of 67 cases where 2D diagnosis was no apparent criteria for MAP diagnosis using the same parameters, eighteen had a hysterectomy due to adherent placenta during the cesarean delivery and were subsequently confirmed to have MAP by histopathology. The rest of them (49 cases) underwent a cesarean delivery only. Of 47 women who had a cesarean hysterectomy, 45 cases $(95.74 \%)$ had confirmed MAP based on the histopathological examination of the hysterectomy specimen; 21 were accreta, 13 increta, and 11 percreta.

\subsection{Prediction of MAP Using 3D Doppler Indices}

Figure 2 and Figure 3 illustrate a 3D analysis of vascular indices and an ultrasound comparison of a placenta with MAP and No MAP respectively.

When the 3D Doppler indices were compared between pregnancies with MAP and those with no MAP, the mean values of VI and VFI were significantly higher in the confirmed MAP group (Table 2; p $<0.001$ both). FI values were similar in both groups, ROC curve was constructed to assess the ability VI, FI, VFI to predict a morbidly adherent placenta and an area under the curve (AUC) was calculated for each parameter (Figure 4). Among the 3D Doppler indices, VI had the highest AUC and FI had the lowest (Table 2, Figure 4) VI $\geq 16$ predicted

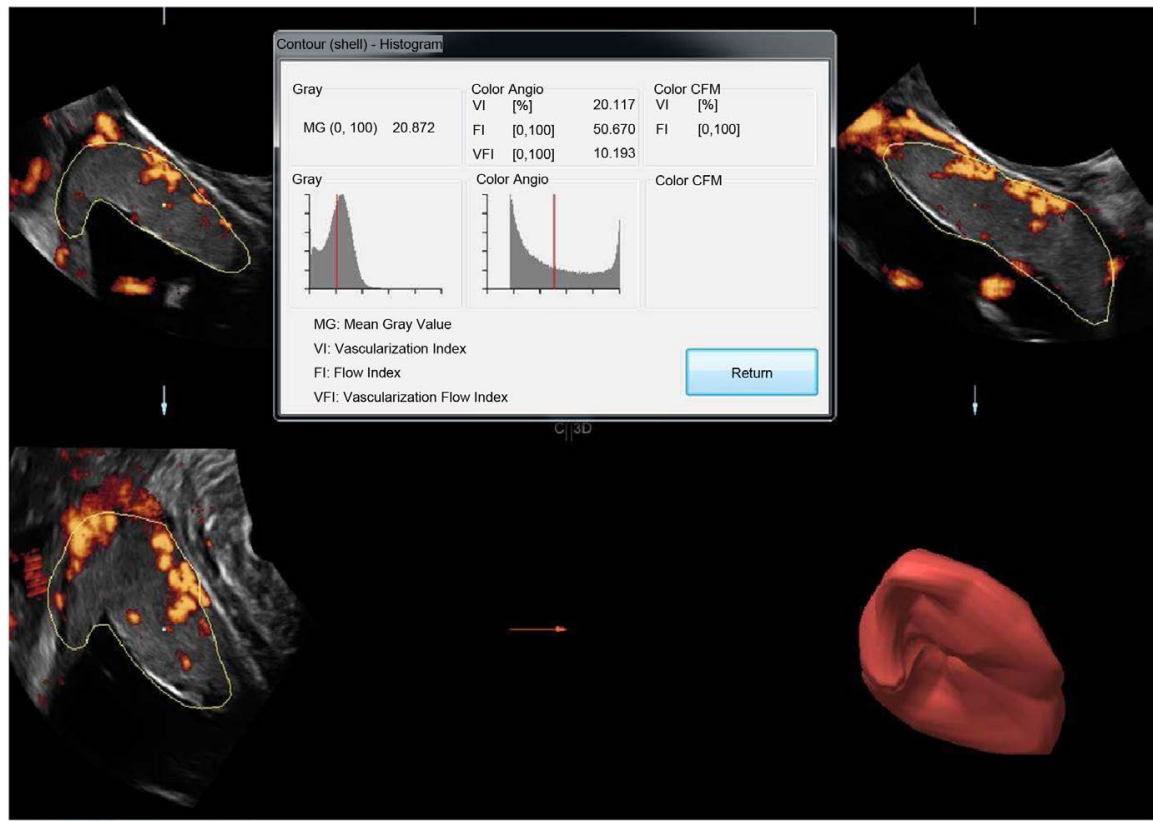

Figure 2. Manual tracing of placental volume at a $30^{\circ}$ angle and calculation of vascular (VI), flow (FI), and vascular flow (VFI) indices. 

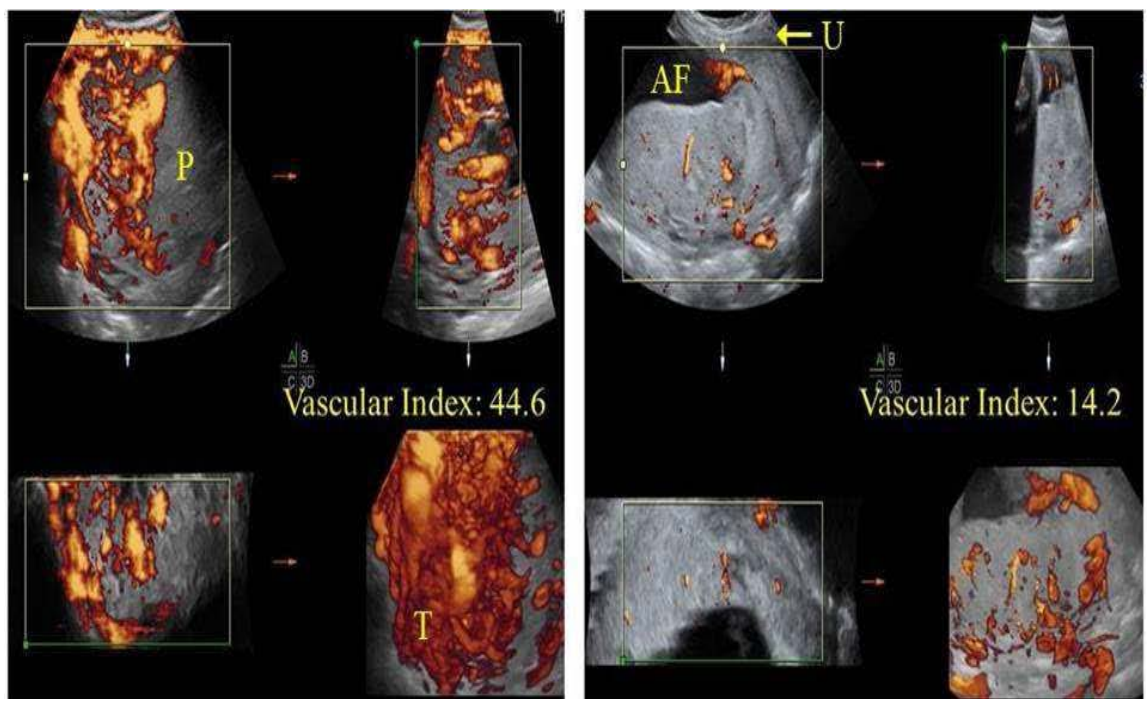

Figure 3. Ultrasound comparison of 3D Doppler placenta between MAP with percreta (left) and No MAP (right), P, placenta; AF, amniotic fluid; U, uterine wall; T, tortuous vessels.

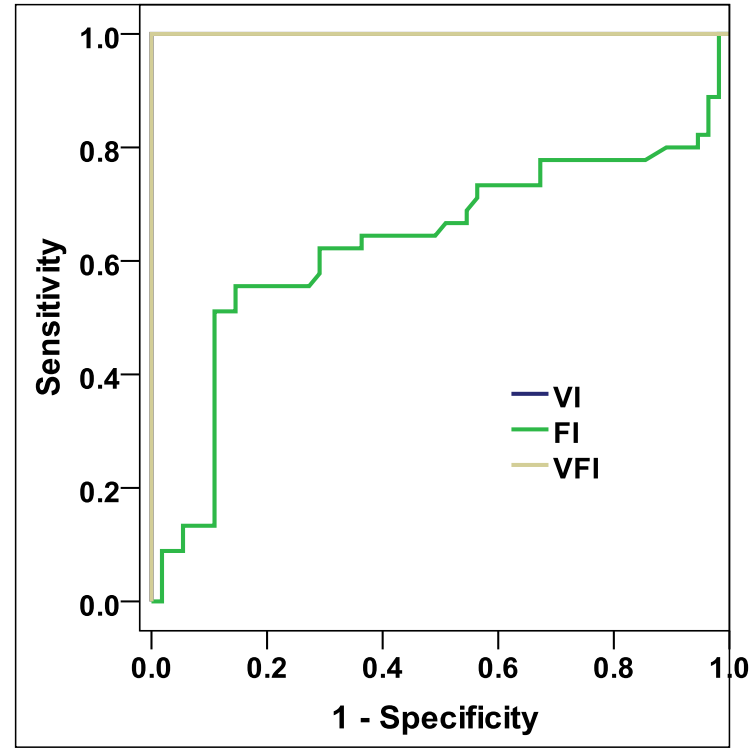

Figure 4. ROC curve in the prediction in differentiating MAP from non-MAP (VI and VFI are overlapping).

the diagnosis of MAP with a $100 \%$ sensitivity and $100.0 \%$ specificity (Table 4 ).

Table 2(a) and Figure 4: VI and VFI had significant perfect diagnostic performance in differentiating MAP from non-MAP. FI had significant low diagnostic performance in differentiating MAP from non-MAP.

\subsection{Prediction of Severe MAP (sMAP) Using 3D Doppler Indices}

sMAP was found in 23/45 (51.2\%) patients. The mean of calculated blood loss volume was on average $3.5 \pm 0.9 \mathrm{~L}$. The placenta pathology in the sMAP was 7 increta and 11 percreta. 
Table 2. AUC: Area under curve, SE: Standard error, CI: Confidence interval, ${ }^{*}$ significant. (a) MAP vs. no MAP. (b) MAP vs. non s MAP.

(a)

\begin{tabular}{cccccc}
\hline Indices & $\mathrm{AUC}$ & $\mathrm{SE}$ & $\mathrm{p}$ & $95 \% \mathrm{CI}$ & Cut off \\
\hline VI & 1.000 & 0.000 & $<0.001^{*}$ & $1.000-1.000$ & $\geq 16.0$ \\
FI & 0.629 & 0.061 & $\mathbf{0 . 0 2 7 ^ { * }}$ & $0.510-0.748$ & $\geq 42.8$ \\
VFI & 1.000 & 0.000 & $<0.001^{*}$ & $1.000-1.000$ & $\geq 11.0$ \\
\hline
\end{tabular}

(b)

\begin{tabular}{cccccc}
\hline Indices & AUC & SE & $\mathrm{p}$ & $95 \%$ CI & Cut off \\
\hline VI & 0.751 & 0.080 & $0.004^{*}$ & $0.595-0.907$ & $\geq 33.1$ \\
FI & 0.662 & 0.083 & 0.063 & $0.500-0.824$ & $\geq 43.0$ \\
VFI & 0.646 & 0.084 & 0.093 & $0.481-0.811$ & $\geq 15.0$ \\
\hline
\end{tabular}

Similarly, using VI, FI, and VFI measured by 3D power Doppler for the prediction of severe MAP. VI of more than 33.1 predicted sMAP with a sensitivity of $73.9 \%$ and $86.4 \%$ specificity (Table 3 ).

ROC curve was used to evaluate the ability VI, FI, VFI to predict (sMAP) and an (AUC) was calculated for each parameter (Table 2, Figure 5). Among the 3D Doppler indices, VI had the highest AUC and VFI had the lowest (Table 2).

Table 2(b) and Figure 5: Only VI had significant moderate diagnostic performance in differentiating sMAP from non-sMAP.

Table 3 shows that: VI $\geq 33.1$ had highest diagnostic characteristics in differentiating sMAP from non-sMAP.

\subsection{Comparing 2D Findings and 3d Findings}

Comparison between obtained data of all the studied cases to assess the best diagnostic method for prediction of MAP and sMAP weather 2D or 3D power Doppler. This comparison is better seen in Table 3 and Table 4. Our study assessed the entire viewed placenta. We found a significant increase of VI in MAP cases in the current study. This strongly suggests that the use of the 3D Doppler vascular index (VI) can accurately predict the diagnosis of morbidly adherent placenta by quantifying the colored voxels within the placental volume indicating an increased vascularity. Furthermore, the values are higher when the clinical severity is increased. The sensitivity and the negative predictive value in predicting sMAP can reach $100 \%$ when a threshold value for VI $\geq 33.1$ is used. Similar increases were seen with VFI. However, we found that FI was not increased in association with MAP.

Table 4: VI $\geq 16.0$ and VFI $\geq 11.0$ had perfect diagnostic characteristics in differentiating MAP from non-MAP.

$2 \mathrm{D}$ and $\mathrm{FI} \geq 42.8$ had significant low diagnostic characteristics in differentiating MAP from non-MAP.

\section{Discussion}

The incidence of MAP has continued to rise primarily due to the increase in 
Table 3. Diagnostic characteristics of $2 \mathrm{D}$ and $3 \mathrm{D}$ indices in differentiating sMAP from non-sMAP.

\begin{tabular}{|c|c|c|c|c|}
\hline \multirow{2}{*}{ Characters } & Value & $95 \% \mathrm{CI}$ & Value & $95 \% \mathrm{CI}$ \\
\hline & \multicolumn{2}{|r|}{$2 \mathrm{D}$} & \multicolumn{2}{|c|}{$\mathrm{VI} \geq 33.1$} \\
\hline Sensitivity & $69.6 \%$ & $47.1 \%-86.8 \%$ & $73.9 \%$ & $51.6 \%-89.8 \%$ \\
\hline Specificity & $50.0 \%$ & $28.2 \%-71.8 \%$ & $86.4 \%$ & $65.1 \%-97.1 \%$ \\
\hline DA & $60.0 \%$ & $44.3 \%-74.3 \%$ & $80.0 \%$ & $65.4 \%-90.4 \%$ \\
\hline Youden's index & $19.6 \%$ & $-8.5 \%-47.7 \%$ & $60.3 \%$ & $37.3 \%-83.2 \%$ \\
\hline PPV & $59.3 \%$ & $38.8 \%-77.6 \%$ & $85.0 \%$ & $62.1 \%-96.8 \%$ \\
\hline NPV & $61.1 \%$ & $35.7 \%-82.7 \%$ & $76.0 \%$ & $54.9 \%-90.6 \%$ \\
\hline LR+ & 1.39 & $0.85-2.29$ & 5.42 & $1.84-15.95$ \\
\hline \multirow[t]{2}{*}{ LR- } & 0.61 & $0.29-1.28$ & 0.30 & $0.15-0.61$ \\
\hline & \multicolumn{2}{|c|}{$\mathrm{FI} \geq 43.1$} & \multicolumn{2}{|c|}{$\mathrm{VFI} \geq 15.0$} \\
\hline Sensitivity & $60.9 \%$ & $38.5 \%-80.3 \%$ & $47.8 \%$ & $26.8 \%-69.4 \%$ \\
\hline Specificity & $68.2 \%$ & $45.1 \%-86.1 \%$ & $81.8 \%$ & $59.7 \%-94.8 \%$ \\
\hline DA & $64.4 \%$ & $48.8 \%-78.1 \%$ & $64.4 \%$ & $48.8 \%-78.1 \%$ \\
\hline Youden's index & $29.1 \%$ & $1.2 \%-56.9 \%$ & $29.6 \%$ & $3.6 \%-55.7 \%$ \\
\hline PPV & $66.7 \%$ & $43.0 \%-85.4 \%$ & $73.3 \%$ & $44.9 \%-92.2 \%$ \\
\hline NPV & $62.5 \%$ & $40.6 \%-81.2 \%$ & $60.0 \%$ & $40.6 \%-77.3 \%$ \\
\hline $\mathrm{LR}+$ & 1.91 & $0.96-3.83$ & 2.63 & $0.98-7.04$ \\
\hline LR- & 0.57 & $0.32-1.03$ & 0.64 & $0.41-0.99$ \\
\hline
\end{tabular}

CI: Confidence interval, DA: Diagnostic accuracy, PPV: Positive Predictive value, NPV: Negative Predictive value, LR+: Positive likelihood ratio, LR-: Negative likelihood ratio.

Table 4. Diagnostic characteristics of $2 \mathrm{D}$ and $3 \mathrm{D}$ in differentiating MAP from no MAP.

\begin{tabular}{|c|c|c|c|c|}
\hline \multirow{2}{*}{ Characters } & Value & $95 \% \mathrm{CI}$ & Value & $95 \% \mathrm{CI}$ \\
\hline & \multicolumn{2}{|c|}{$2 \mathrm{D}$} & \multicolumn{2}{|c|}{$\mathrm{VI} \geq 16.0$} \\
\hline Sensitivity & $60.0 \%$ & $44.3 \%-74.3 \%$ & $98.1 \%$ & $89.1 \%-99.3 \%$ \\
\hline Specificity & $89.1 \%$ & $77.8 \%-95.9 \%$ & $96.3 \%$ & $93.5 \%-98.6 \%$ \\
\hline DA & $76.0 \%$ & $66.4 \%-84.0 \%$ & $97.4 \%$ & $96.4 \%-100.0 \%$ \\
\hline Youden's index & $49.1 \%$ & $32.6 \%-65.6 \%$ & $94.4 \%$ & $91.5 \%-97.5 \%$ \\
\hline PPV & $81.8 \%$ & $64.5 \%-93.0 \%$ & $93.12 \%$ & $85.5 \%-96.3 \%$ \\
\hline NPV & $73.1 \%$ & $60.9 \%-83.2 \%$ & $90.7 \%$ & $80.9 \%-94.3 \%$ \\
\hline $\mathrm{LR}+$ & 5.50 & $2.49-12.14$ & 11.45 & $8.06 \%-13.75 \%$ \\
\hline \multirow[t]{2}{*}{ LR- } & 0.45 & $0.31-0.65$ & 0.05 & $0.03-0.13$ \\
\hline & \multicolumn{2}{|c|}{$\mathrm{FI} \geq 42.8$} & \multicolumn{2}{|c|}{$\mathrm{VFI} \geq 11.0$} \\
\hline Sensitivity & $62.2 \%$ & $46.5 \%-76.2 \%$ & $98.1 \%$ & $89.1 \%-99.3 \%$ \\
\hline Specificity & $70.9 \%$ & $57.1 \%-82.4 \%$ & $96.3 \%$ & $93.5 \%-98.6 \%$ \\
\hline $\mathrm{DA}$ & $67.0 \%$ & $56.9 \%-76.1 \%$ & $97.4 \%$ & $96.4 \%-100.0 \%$ \\
\hline Youden's index & $33.1 \%$ & $14.6 \%-51.7 \%$ & $94.4 \%$ & $91.5 \%-97.5 \%$ \\
\hline PPV & $63.6 \%$ & $47.8 \%-77.6 \%$ & $93.12 \%$ & $85.5 \%-96.3 \%$ \\
\hline NPV & $69.6 \%$ & $55.9 \%-81.2 \%$ & $90.7 \%$ & $80.9 \%-94.3 \%$ \\
\hline $\mathrm{LR}+$ & 2.14 & $1.34-3.43$ & 11.45 & $8.06 \%-13.75 \%$ \\
\hline LR- & 0.53 & $0.35-0.80$ & 0.05 & $0.03-0.13$ \\
\hline
\end{tabular}

CI: Confidence interval, DA: Diagnostic accuracy, PPV: Positive Predictive value, NPV: Negative Predictive value, LR+: Positive likelihood ratio, LR-: Negative likelihood ratio. 


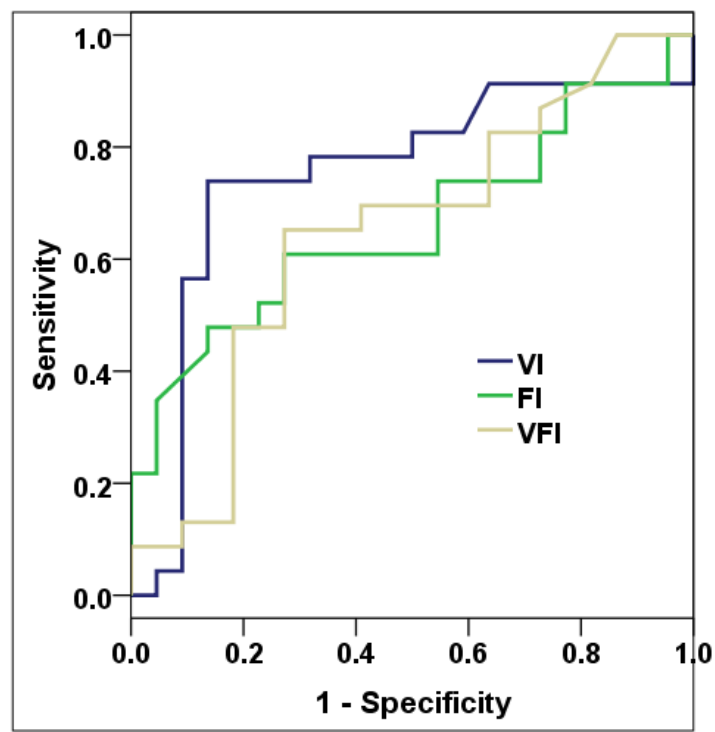

Figure 5. ROC curve in differentiating sMAP from non-sMAp.

cesarean delivery rates with an overall incidence of approximately 1 in 533 pregnancies [3]. MAP can result in major maternal morbidities such as life-threatening hemorrhage and intra-operative organ injuries [16]. Therefore, it is important to be able to detect this entity antenatally with high reliability to allow for a proper preparation for delivery in an appropriate unit equipped to handle potentially complicated surgery [17] [18].

The diagnosis of MAP has traditionally been suspected on 2D ultrasound and color Doppler. Although how the color Doppler findings should be used is subjective and the published descriptions of exactly what findings are predictive of MAP are not specific, Color Doppler of vascular patterns in the placenta and MRI may also be helpful in establishing the diagnosis [2].

Despite these reported findings, the diagnosis of MAP remains as a subjective diagnosis per the interpretation of the observer [8]. Additionally, there are limited previously published studies available for $2 \mathrm{D}$ to determine the severe form of MAP that is associated with massive hemorrhage, percreta or increta, and intensive care admission.

During the last few years, three-dimensional (3D) power Doppler technique was introduced as a tool to diagnose MAP based again on the observer's interpretation of 3D images. The objective of our study was to analyze the ability of gray-scale two-dimensional (2D) ultrasound vs. 3D power Doppler vascular and flow indices to objectively predict MAP and severe MAP. We hypothesized that the calculation of 3D power Doppler indices can accurately predict MAP and severe MAP.

\subsection{Findings and Interpretation}

Our study assessed the entire viewed placenta. We found a significant increase of 
VI in MAP cases in the current study. This strongly suggests that the use of the 3D Doppler vascular index (VI) can accurately predict the diagnosis of morbidly adherent placenta by quantifying the colored voxels within the placental volume indicating an increased vascularity with a $100 \%$ sensitivity and $100.0 \%$ specificity. Furthermore, the indices are higher when the clinical severity is increased. The sensitivity of $73.9 \%, 86.4 \%$ specificity and the negative predictive value in predicting sMAP was $90.7 \%$ when a threshold value for VI of greater than 33.1 is used. Similar increases were seen with VFI. However, we found that FI was not increased in association with MAP.

In the current study the sensitivity and the specificity of $2 \mathrm{D}$ ultrasound for the diagnosis of MAP was $60.0 \%$ and $89.1 \%$ respectively when at least one abnormal parameter was present. The sensitivity and specificity dropped to $69.6 \%$ and $50.0 \%$ respectively when severe MAP was the outcome.

There is a significant difference in the ability of prenatal prediction of MAP and sMAP between 2D ultrasound and 3D power Doppler which state that the 3D power Doppler is a more accurate diagnostic tool of MAP than traditional 2D ultrasound.

\subsection{Comparison with Previous Studies}

Previously reported studies to diagnose MAP by $2 \mathrm{D}$ ultrasound have concluded a range between $76 \%-94 \%$ and $74 \%-99 \%$ for sensitivity and specificity respectively [2] [18] [19] [20]. This wide range is probably attributed to the subjectivity of the $2 \mathrm{D}$ parameters and the variation in sample size.

Another factor that affected this range was the review by a single examiner. When multiple examiners blindly reviewed every ultrasound examination, the sensitivity and the specificity were found to be even lower (63\% and $87 \%$ respectively) [21]. There was a systematic review showed a wide heterogeneity in terminology used to describe the grades of accreta placentation using the $2 \mathrm{D}$ parameters [22]. The authors concluded that the evaluation of the accuracy of ultrasound imaging in screening and diagnosing placenta accreta is limited [22].

All these factors were avoided in our study by using the latest and the most accurate description of 2D findings, considerable sample size and the examination was reviewed by single examiner for $2 \mathrm{D}$ ultrasound and another for $3 \mathrm{D}$ power Doppler.

Several studies used the 3D color Doppler technique to diagnose MAP and reported a sensitivity ranging between $39 \%$ and $100 \%$ based on abnormal vascular patterns [8] [23] [24]. In these studies, the placenta was assessed subjectively for abnormal vascularity using the 3D power Doppler technique without any quantification.

Another study quantified the amount of vessels involved in the diagnosis of morbidly adherent placenta [9]. However, this was applied to the utero-placental interface only and not the entire placenta. They measured the largest area of confluent 3D power Doppler signal [9]. Although abnormal vascularity of the 
utero-placenta interface is highly suggestive of abnormal placentation, abnormal vessels running throughout the placenta could be missed using this particular technique. In addition, choosing the most confluent area is operator dependent and other areas might have been missed due to subjectivity of the operator's assessment. While in our study the placenta was traced manually with examination of vascularity of placenta and utero-vesicle space and at the maximum placental thickness using offline analysis of an optimized 3d Doppler images and the vascular indices were measured to decrease the percentage of subjective operator's error.

In Abdel Moniem et al. [25] study on 50 pregnant patients with suspected MAP, using both 2D US and 3D Power Doppler scans to assess the best parameter that could predict MAP prenatally. The results show that the best $2 \mathrm{D}$ parameter was disruption of uterine serosal interface (81.8 sensitivity), so could be used in screening for MAP and the best parameter for confirmation of MAP diagnosis was presence of exophytic mass invading the bladder with $94.9 \%$ specificity.

While the best 3D power Doppler parameter detected was disruption of uterine serosal interface ( 90.9 sensitivity and $68.8 \%$ specificity). this study offer the $3 \mathrm{D}$ power Doppler as a complementary tool to $2 \mathrm{D}$ us in diagnosis of MAP which is not in line with our study that present $3 \mathrm{D}$ power Doppler as separate accurate diagnostic tool with definitive criteria that is not subjective to the interpretation of the examiner.

The same concept was used in the study conducted by Hussein et al. which also agreed to the use of 3D power Doppler with both 2D-US and color Doppler as complementary techniques could improve the antenatal diagnosis or exclusion of morbidly adherent placenta, but ended with the same subjective findings that could easily misinterpreted by sonographers.

While in Haidar et al. [10] study conducted to 50 women examined using 2D US and 3D power Doppler that measures placental vascular indices using VOCAL software applied to analyze offline placental images. it stated that vascular index of more than 21 accurately predicts the morbidly adherent placenta in patients with placenta previa with a sensitivity and specificity of $95 \%$ and $92 \%$ respectively, which is similar to our results but MAP was predicted at VI $\geq 16$ which have better sensitivity and specificity values (100\% both) In addition, compared to 2D ultrasound; 3D Power Doppler vascular (more than 31) and vascular flow indices were more predictive diagnostic tools of severe MAP with a sensitivity of $100 \%$ and specificity of $90 \%$. As for our results it shows a higher cut off value of VI to predict sMAP ( $\geq 33.1)$ with much less sensitivity of $73.9 \%$ and less specificity of 86.4. But this study has a low sample size of 50 cases which is not enough to generalize the results and used VOCAL software offline analysis of placental images of maximum placental thickness using 4D view ,such technology is not available to all maternity centers. Despite the differences between this study and our study both concluded that vascular index accurately predicts 
the morbidly adherent placenta in patients with placenta previa. In addition, 3D Power Doppler vascular and vascular flow indices were more predictive of severe cases of MAP compared to 2D ultrasound.

\subsection{Clinical Implications}

Clinically, the use of VI can detect a number of cases with invasive placentation that the conventional 2D ultrasound cannot, which allows for a better preparation for surgery.

Despite the limitations, the major strength of our study is the objective nature of the 3D Doppler technique for the diagnosis of MAP. The technique has a short learning curve and is reproducible. It has the potential to reduce the variation between examiners and increase the rate of antenatal detection of MAP leading to a decrease in maternal and fetal morbidities.

\subsection{Weaknesses and Strengths}

The small sample size is one of the limitations of our study. In addition, only women with placenta previa were included as we considered this a risk factor for MAP that would allow us to have a better yield for this disease in our study population. MAP may also occur in women without placenta previa; however our study did not address this population. Our study period was limited to an interval of gestational age of 28 - 32 weeks and diagnosing the condition prior to 28 weeks gestation needs to be studied further. We also could not adjust for other factors such as the number of previous $\mathrm{CD}$ due to the sample size. Additionally, 3D color Doppler volume studies are currently available only in the GE Voluson machines.

This may not apply to other technologies and with changing technology the indices may change for the diagnosis, which will need to be revalidated. Our study did not assess MRI ability to predict MAP in comparison to 3D Doppler indices. However, MRI is more expensive and less readily available.

Despite the limitations, the major strength of our study is the objective nature of the 3D Doppler technique for the diagnosis of MAP. The technique has a short learning curve and is reproducible. It has the potential to reduce the variation between examiners and increase the rate of antenatal detection of MAP leading to a decrease in maternal and fetal morbidities.

\section{Conclusions}

In this study, our results showed that the $3 \mathrm{D}$ color Doppler VI $\geq 16$ predicted the diagnosis of MAP with a $100 \%$ sensitivity, $100 \%$ specificity which are better than those of $2 \mathrm{D}$ ultrasound ( $60.0 \%$ and $89.1 \%$ respectively).

Severe MAP occurred in 51.2\% of MAP and 3D color Doppler of VI $>33.1$ predicted severe MAP with a sensitivity of $73.9 \%$ and specificity of $86.4 \%$, which was superior to $2 \mathrm{D}$ ultrasound.

In the current study, the sensitivity and the specificity of $2 \mathrm{D}$ ultrasound for 
the diagnosis of MAP were $60.0 \%$ and $89.1 \%$ respectively when at least one abnormal parameter was present. The sensitivity and specificity dropped to $69.6 \%$ and $50.0 \%$ respectively when severe MAP was the outcome.

\section{Future Research Implications}

$3 \mathrm{D}$ power Doppler is introduced as an objective technique that may limit the variations in diagnosing MAP due to the subjectivity of $2 \mathrm{D}$ ultrasound interpretations. So, a multi-center prospective study is needed to validate our findings, and confirm the usefulness of this methodology and its ability to improve the prenatal diagnosis of this condition compared to 2D ultrasound before an appropriate clinical application is determined.

Other available imaging technologies such as placental elastography and 3D MSV Doppler should be assessed to measure these indices with a larger sample size to design definitive prenatal diagnostic measures of MAP to decrease maternal and fetal morbidity and mortality.

\section{Conflicts of Interest}

The authors declare no conflicts of interest regarding the publication of this paper.

\section{References}

[1] Hui, P. (2011) Developmental Biology of the Placenta. In: Hui, P., Ed., Gestational Trophoblastic Disease, Springer, Berlin, 15-39. https://doi.org/10.1007/978-1-61779-394-3 2

[2] Abuhamad, A. (2013) Morbidly Adherent Placenta. Seminars in Perinatology, 37, 359-364. https://doi.org/10.1053/j.semperi.2013.06.014

[3] Silver, R. (2015) Abnormal Placentation. Obstetrics \& Gynecology, 126, 654-668. https://doi.org/10.1097/AOG.0000000000001005

[4] Fox, H. and Sebire, N. (2007) Pathology of the Placenta. Saunders Elsevier, London. https://doi.org/10.1016/B978-1-4160-2592-4.50018-7

[5] Jauniaux, E. and Grobman, W.A. (2016) Textbook of Caesarean Section. Oxford University Press, Oxford. https://doi.org/10.1093/med/9780198758563.003.0001

[6] Jauniaux, E. and Jurkovic, D. (2012) Placenta Accreta: Pathogenesis of a 20th Century Iatrogenic Uterine Disease. Placenta, 33, 244-251. https://doi.org/10.1016/j.placenta.2011.11.010

[7] Danforth, D.N. and Scott, J.R. (2003) Danforth's Obstetrics and Gynecology. 9th Edition, Lippincott Williams \& Wilkins, Philadelphia, Ch. 20.

[8] Shih, J.C., Palacios Jaraquemada, J.M., Su, Y.N., et al. (2009) Role of Three-Dimensional Power Doppler in the Antenatal Diagnosis of Placenta Accreta: Comparison with Gray-Scale and Color Doppler Techniques. Ultrasound in Obstetrics \& Gynecology, 33, 193-203. https://doi.org/10.1002/uog.6284

[9] Chou, M.M., Chen, W.C., Tseng, J.J., Chen, Y.F., Yeh, T.T. and Ho, E.S. (2009) Prenatal Detection of Bladder Wall Involvement in Invasive Placentation with Sequential Two-Dimensional and Adjunctive Three-Dimensional Ultrasonography. Taiwanese Journal of Obstetrics \& Gynecology, 48, 38-45. https://doi.org/10.1016/S1028-4559(09)60033-4 
[10] Haidar, Z.A., Papanna, R., Sibai, B.M., Tatevian, N., Viteri, O.A., Vowels, P.C., Blackwell, S.C. and Moise, J.R. (2017) Can 3D Power Doppler Indices Improve the Prenatal Diagnosis of a Potentially Morbidly Adherent Placenta in Patients with Placenta Previa? American Journal of Obstetrics and Gynecology, 217, 202.e1-202.e13. https://doi.org/10.1097/01.aoa.0000532304.98988.bc

[11] Hafner, E., Metzenbauer, M., Stümpflen, I., Waldhör, T. and Phili, K. (2010) First Trimester Placental and Myometrial Blood Perfusion Measured by 3D Power Doppler in Normal and Unfavourable Outcome Pregnancies. Placenta, 31, 756-763. https://doi.org/10.1016/j.placenta.2010.06.011

[12] Gonzalez, N.L., Gonzalez Davila, E., Castro, A., Padron, E. and Plasencia, W. (2014) Effect of Pregestational Diabetes Mellitus on First Trimester Placental Characteristics: Three-Dimensional Placental Volume and Power Doppler Indices. Placenta, 35, 147-151. https://doi.org/10.1016/j.placenta.2014.01.002

[13] Rath, W.H. (2011) Postpartum Hemorrhage-Update on Problems of Definitions and Diagnosis. Acta Obstetricia et Gynecologica Scandinavica, 90, 421-428. https://doi.org/10.1111/j.1600-0412.2011.01107.x

[14] Carroli, G., et al. (2008) Epidemiology of Postpartum Haemorrhage: A Systematic Review. Best Practice \& Research Clinical Obstetrics \& Gynaecology, 22, 999-1012. https://doi.org/10.1016/j.bpobgyn.2008.08.004

[15] McHugh, M.L. (2012) Interrater Reliability: The Kappa Statistic. Biochemiamedica, 22, 276-282. https://doi.org/10.11613/BM.2012.031

[16] Creanga, A.A., Bateman, B.T., Butwick, A.J., Raleigh, L., Maeda, A., Kuklina, E. and Callaghan, W.M. (2015) Morbidity Associated with Cesarean Delivery in the United States: Is Placenta Accreta an Increasingly Important Contributor? American Journal of Obstetrics \& Gynecology, 213, 384.e1-11. https://doi.org/10.1016/j.ajog.2015.05.002

[17] Bhide, A., Sebire, N., Abuhamad, A., Acharya, G. and Silver, R. (2017) Morbidly Adherent Placenta: The Need for Standardization. Ultrasound in Obstetrics \& Gynecology, 49, 559-563. https://doi.org/10.1002/uog.17417

[18] D’Antonio, F., Iacovella, C., Jaraquemada, P., Bruno, C.H., Manzoli, L. and Bhide, A. (2014) Prenatal Identification of Invasive Placentation Using Magnetic Resonance Imaging: Systematic Review and Meta-Analysis. Ultrasound in Obstetrics \& Gynecology, 44, 8-16. https://doi.org/10.1002/uog.13327

[19] Pilloni, E., Alemanno, M.G., Gaglioti, P., Sciarrone, A., Garofalo, A., Biolcati, M., Botta, G., et al. (2016) The Accuracy of Ultrasound in Antenatal Diagnosis of Placental Attachment Disorders. Ultrasound in Obstetrics \& Gynecology, 47, 302-307. https://doi.org/10.1002/uog.14893

[20] Rezk, M.A. and Shawky, M. (2016) Grey-Scale and Colour Doppler Ultrasound versus Magnetic Resonance Imaging for the Prenatal Diagnosis of Placenta Accreta. The Journal of Maternal-Fetal \& Neonatal Medicine, 29, 218-223. https://doi.org/10.3109/14767058.2014.993604

[21] Bowman, Z.S., Eller, A.G., Kennedy, A.M., Richards, D.S., Winter, T.C., Woodward, P.J. and Silver, R.M. (2014) Accuracy of Ultrasound for the Prediction of Placenta Accreta. American Journal of Obstetrics and Gynecology, 211, 177.e1-7. https://doi.org/10.1016/j.ajog.2014.03.029

[22] Jauniaux, E., Collins, S.L., Jurkovic, D. and Burton, G.J. (2016) Accreta Placentation: A Systematic Review of Prenatal Ultrasound Imaging and Grading of Villous Invasiveness. American Journal of Obstetrics and Gynecology, 215, 712-721. https://doi.org/10.1016/j.ajog.2016.07.044 
[23] Calì, G., Giambanco, L., Puccio, G. and Forlani, F. (2013) Morbidly Adherent Placenta: Evaluation of Ultrasound Diagnostic Criteria and Differentiation of Placenta Accreta from Percreta. Ultrasound in Obstetrics \& Gynecology, 41, 406-412. https://doi.org/10.1002/uog.12385

[24] Collins, S.L., Ashcroft, A., Braun, T., Calda, P., Langhoff-Roos, J., Morel, O., Stefanovic, V., et al. (2016) Proposal for Standardized Ultrasound Descriptors of Abnormally Invasive Placenta (AIP). Ultrasound in Obstetrics \& Gynecology, 47, 271-275. https://doi.org/10.1002/uog.14952

[25] Abdel Moniem, A.M., et al. (2015) Accuracy of Three-Dimensional Multislice View Doppler in Diagnosis of Morbid Adherent Placenta. Journal of the Turkish-German Gynecological Association, 16, 126-136. https://doi.org/10.5152/jtgga.2015.15038 